\title{
Analysis and Experimental Evaluation of the Intrinsically Passive Controller (IPC) for Multifingered Hands
}

\author{
Thomas Wimböck*, Christian Ott ${ }^{\dagger}$ and Gerd Hirzinger* \\ *Institute of Robotics and Mechatronics, German Aerospace Center (DLR), Germany, Email: thomas.wimboeck@dlr.de \\ ${ }^{\dagger}$ Department of Mechano-Informatics, University of Tokyo, Japan
}

\begin{abstract}
The object level control of a dexterous robot hand provides an intuitive high-level interface to solve fine manipulation tasks. In the past, many algorithms were proposed based on a weighted pseudoinverse of the grasp map. In a different approach Stramigioli introduces a virtual object called "Intrinsically Passive Controller (IPC)". This controller distributes the generalized object forces using coupling springs whose weighting have an intuitive physical meaning. Even though this controller has been known for several years we will present the first experimental results for a four-fingered hand. Furthermore, the term virtual grasp map is introduced and a method to parameterize the stiffness parameters in order to obtain an effective object level stiffness and a damping design is proposed. An implementation of the IPC is tested on the DLR Hand II and its performance is analyzed by manipulating soft and stiff objects.
\end{abstract}

\section{INTRODUCTION}

Advanced manipulation skills gained recently more and more attention. In the past, the capabilities of humanoid robots concentrated on walking. Lately, there has been a large interest in equipping them with advanced manipulation skills. Such robots with dexterous arms and hands are very flexible and can perform many different tasks like manipulation of various objects (e.g. large and heavy or small and fragile) or can gesticulate. A dexterous robot hand possesses usually many $\mathrm{DOF}^{1}$ for which controllers with joint or Cartesian command interface are used. Object level control is employed if the task is to fine manipulate an object. It has in general the advantages that it is easy to define grasp forces, to compensate for robot and object inertia, to specify external forces acting on the object and to avoid unnecessary high internal forces [1]. A general overview of the control of dexterous hands can be found in [2].

Object impedance is realized by a stiffness which is defined by the reaction of the object frame $\boldsymbol{H}_{r}$ to an externally applied generalized force $\boldsymbol{w}_{\text {ext }}$. In contrast to joint or Cartesian ${ }^{2}$ level impedance control the direct kinematics $\boldsymbol{H}_{r}$ cannot be determined easily. The object pose estimation by cameras is still a tough problem due to the occlusion by the manipulator. Other methods to determine the current object frame, are the simulation of the object dynamics, the integration of the Cartesian fingertip velocity over time or the use of virtual objects. The use of virtual objects has been presented by several authors [3], [4]. The concepts of grasp

\footnotetext{
${ }^{1}$ DOF - degree of freedom.

${ }^{2}$ Considering serial kinematics, e. g. with respect to a single fingertip or to the wrist of a robot arm.
}

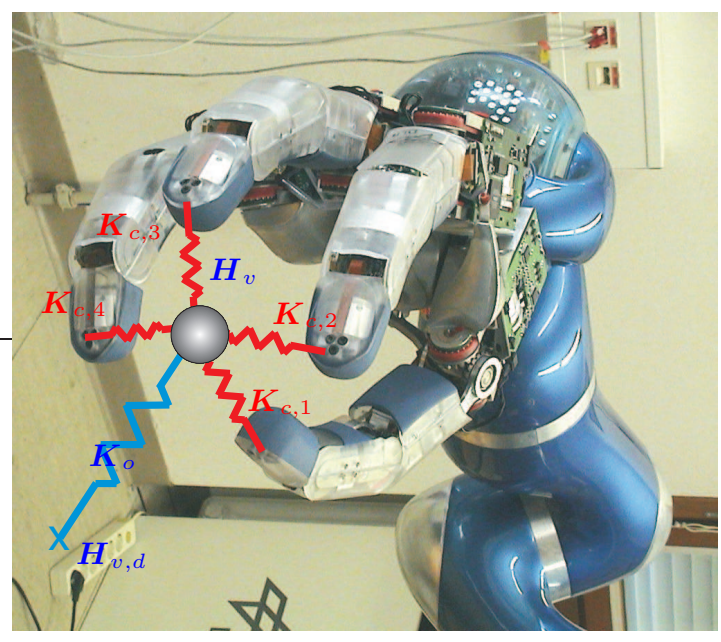

Fig. 1. DLR Hand II superimposed by the virtual springs defined by the potential functions in equation (10) and the virtual object.

force realization can be divided into impedance control [3], [5]-[7] and force control [1], [8], [9]. The main disadvantage of force control is the requirement of contact. A force control strategy is only meaningful during contact, hence it is not suited to handle the change between contact and non-contact [3]. The impedance controller has the advantage that stability is given independently of the contact state since it will converge to an equilibrium state that is the desired position in case of free motion and that is a stable equilibrium position in case of interaction with a passive environment. As a consequence, only impedance-based approaches are treated in the following. This fact facilitates the stability analysis and the usability of the controller. In previous works we described a passivity-based object level impedance controller for a multifingered hand [10] which we used in [6] to develop a two-handed impedance behavior for DLR's humanoid manipulator Justin [11]. The goal of this work is to analyze the Intrinsically Passive Controller (IPC) proposed by Stramigioli [3] and to evaluate the performance of an implementation of this controller through experiments, which were run for the first time on a four-fingered hand, the DLR Hand II [12] (Fig. 1). Therefore, the term of a virtual grasp matrix will be defined and its necessary properties will be given. Furthermore, we propose a damping design as a function of the inertia of the virtual and the real object, the stiffness parameters, and the grasp matrices. 


\section{System Model And Assumptions}

The considered dynamical model for an object and a manipulator with $M$ degrees of freedom has the form of a rigid multi-body-system and is written as [13]

$$
\begin{array}{r}
\boldsymbol{M}_{r o}\left(\boldsymbol{x}_{r}\right) \ddot{\boldsymbol{x}}_{r}+\boldsymbol{C}_{r o}\left(\boldsymbol{x}_{r}, \dot{\boldsymbol{x}}_{r}\right) \dot{\boldsymbol{x}}_{r}+\boldsymbol{g}_{r o}\left(\boldsymbol{x}_{r}\right)=\boldsymbol{w}_{e x t}+\boldsymbol{w}_{g} \\
\boldsymbol{M}_{h}(\boldsymbol{q}) \ddot{\boldsymbol{q}}+\boldsymbol{C}_{h}(\boldsymbol{q}, \dot{\boldsymbol{q}}) \dot{\boldsymbol{q}}+\boldsymbol{g}_{h}(\boldsymbol{q})=\boldsymbol{\tau}+\boldsymbol{\tau}_{e x t}
\end{array}
$$

where $\boldsymbol{q}=\left[\boldsymbol{q}_{f_{1}}, \ldots, \boldsymbol{q}_{f_{N}}\right] \in \mathbb{R}^{M}$ is the vector of generalized positions for $N$ fingers in the hand. The vector $\tau \in \mathbb{R}^{M}$ contains the corresponding generalized actuator forces $^{3}$, which are considered as the control inputs. The vector $\boldsymbol{x}_{r} \in \mathbb{R}^{6}$ is the local representation of the object frame $\boldsymbol{H}_{r} \in S E(3)$. The matrices $\boldsymbol{M}_{h}(\boldsymbol{q}) \in \mathbb{R}^{M \times M}, \boldsymbol{M}_{\text {ro }}\left(\boldsymbol{x}_{r}\right) \in$ $\mathbb{R}^{6 \times 6}$ are the symmetric and positive definite inertia matrices, $\boldsymbol{C}_{h}(\boldsymbol{q}, \dot{\boldsymbol{q}}) \dot{\boldsymbol{q}} \in \mathbb{R}^{M}, \boldsymbol{C}_{r o}\left(\boldsymbol{x}_{r}, \dot{\boldsymbol{x}}_{r}\right) \dot{\boldsymbol{x}}_{r} \in \mathbb{R}^{6}$ contain the centrifugal and Coriolis components, and $\boldsymbol{g}(\boldsymbol{q}) \in \mathbb{R}^{M}, \boldsymbol{g}\left(\boldsymbol{x}_{r_{o}}\right) \in$ $\mathbb{R}^{6}$ are the vectors of generalized gravity forces each for the hand (index $h$ ) and the object (index ro), respectively. Finally, $\boldsymbol{w}_{\text {ext }} \in \mathbb{R}^{6}$ contains external generalized forces acting on the object. The vector $\boldsymbol{w}_{g} \in \mathbb{R}^{6}$ describes the effects of the fingertip forces applied to the object at the contact points. The vector $\tau_{\text {ext }}$ represents the generalized external forces acting on a finger including the contact forces. In order to focus the presentation on the multi-body part of the dynamics, other physical effects like link and joint flexibility as well as joint friction are neglected. In fact, in the experiment in Section IV an underlying torque controller is employed which compensates the joint friction partially.

In this paper the following definitions are used to facilitate the notation. A frame $\boldsymbol{H}_{x}=\left[\boldsymbol{R}_{x}, \boldsymbol{p}_{x}\right] \in S E(3)$ consists of a rotation $\boldsymbol{R}_{x} \in S O(3)$ and a translation $\boldsymbol{p}_{x} \in \mathbb{R}^{3}$. A frame $\boldsymbol{H}_{x}$ can be described by a local parametrization $\boldsymbol{x}_{x} \in$ $\mathbb{R}^{6}$. The vector $\boldsymbol{w}_{x}=\left(\boldsymbol{f}_{x}^{T} \boldsymbol{m}_{x}^{T}\right)^{T}$ contains the generalized forces acting at the coordinate system $\boldsymbol{H}_{x}$ with $\boldsymbol{f}_{x}, \boldsymbol{m}_{x} \in \mathbb{R}^{3}$ represented in the body frame [13].

In the following, fine manipulation with a multifingered hand is treated. Therefore, it is assumed that the $N$ fingertips of each hand are in contact with the object. For point contacts with friction (PCWF), the held object can be manipulated in 3D-space if $N \geq 3$ and if each finger has a configuration space of dimension $\geq 3$. We assume furthermore that

1) The internal forces provided by the controller are chosen to be sufficient such that the friction constraints are fulfilled for all contact points (no sliding).

2) In order to allow $6 \mathrm{D}$ object motion the contacts between the object and the hand are restricted to the fingertips (no palm contact).

3) The relative contact points between the fingertips and the object do not change (neglecting rolling effects).

The $i^{\text {th }}$ Cartesian fingertip position $\boldsymbol{p}_{f_{i}}\left(\boldsymbol{q}_{f_{i}}\right) \in R R^{3}$ and its orientation relative to the inertial frame $\boldsymbol{R}_{f_{i}}\left(\boldsymbol{q}_{f_{i}}\right)$ can be calculated as a function of the generalized positions $\boldsymbol{q}_{f_{i}}$. We can define the hand Jacobian $\boldsymbol{J}_{h}(\boldsymbol{q})=\frac{\partial \boldsymbol{p}_{f}(\boldsymbol{q})}{\partial \boldsymbol{q}^{T}}$ that maps

\footnotetext{
${ }^{3}$ Depending on the type of joint (rotational or prismatic) this generalized force is either a torque or a force.
}

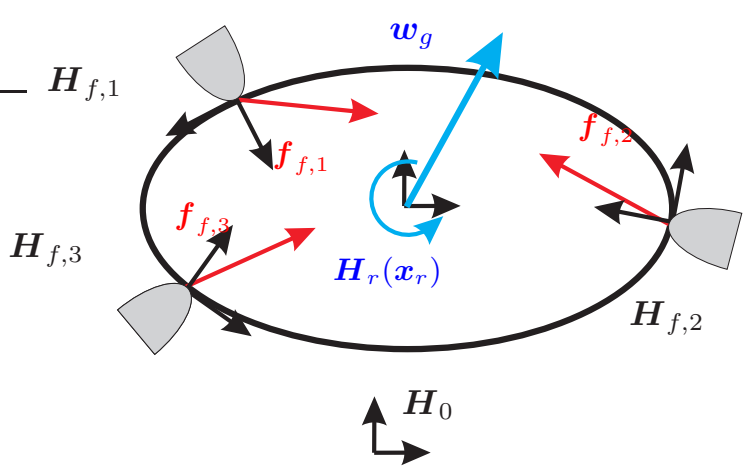

Fig. 2. Visualization of the grasp map $\boldsymbol{G}_{r}$.

the joint velocities to the Cartesian fingertip velocities. The forces $\boldsymbol{f}_{c, i}, \boldsymbol{f}_{f, i}$, the velocities $\dot{\boldsymbol{p}}_{c, i}, \dot{\boldsymbol{p}}_{f, i}$ and the variations of position $\delta \boldsymbol{p}_{c, i}, \delta \boldsymbol{p}_{f, i}(\boldsymbol{q})$ at the $N$ fingertips are used in stacked notation (c.f. Fig. 4). To indicate stacked notation the index is removed (e. g. $\boldsymbol{f}_{c}=\left(\boldsymbol{f}_{c, 1}^{T} \cdots \boldsymbol{f}_{c, N}^{T}\right)^{T}$ ). Note, that these variables are expressed in the inertial frame.

The grasp map $\boldsymbol{G}_{r}$ is used to determine the effect of the stacked contact forces at the fingertips $\boldsymbol{f}_{f} \in \mathbb{R}^{3 N}$ on the object wrench $\boldsymbol{w}_{g}$ (see Fig. 2).

$$
\begin{aligned}
\boldsymbol{G}_{r} & =\left[A d_{\boldsymbol{H}_{r, f_{1}}^{-1}}^{T} \boldsymbol{B} \cdots A d_{\boldsymbol{H}_{r, f_{N}}^{T-1}}^{T} \boldsymbol{B}\right] \boldsymbol{R}_{f}^{T}, \\
\boldsymbol{R}_{f} & =\operatorname{blockdiag}\left\{\boldsymbol{R}_{f_{1}}, \ldots, \boldsymbol{R}_{f_{N}}\right\} \\
\boldsymbol{B} & =\left[\mathrm{I}_{3 \times 3} \mathbf{0}_{3 \times 3}\right]^{T},
\end{aligned}
$$

where $\boldsymbol{B} \in \mathbb{R}^{6 \times 3}$ is the wrench basis which is used to model the point contact with friction [13]. The matrix $\boldsymbol{H}_{r, f_{i}}$ is the configuration of the $i^{\text {th }}$ contact frame relative to the object frame. The additional rotation $\boldsymbol{R}_{f_{i}}^{T}$ transforms the forces and velocities at the fingertips represented in the inertial system into the $i^{t h}$ contact frame. The grasp map relates forces and velocities on Cartesian fingertip level with the ones on object level:

$$
\boldsymbol{w}_{g}=\boldsymbol{G}_{r} \boldsymbol{f}_{f}, \quad \boldsymbol{G}_{r}^{T} \dot{\boldsymbol{x}}_{r}=\dot{\boldsymbol{p}}_{f}, \quad \boldsymbol{G}_{r}^{T} \delta \boldsymbol{x}_{r}=\delta \boldsymbol{p}_{f} .
$$

With the introduced variables the well known grasp constraint ${ }^{4}$ can be formulated as

$$
\boldsymbol{J}_{h}(\boldsymbol{q}) \dot{\boldsymbol{q}}=\boldsymbol{G}_{r}^{T}\left(\boldsymbol{q}, \boldsymbol{x}_{r}\right) \dot{\boldsymbol{x}}_{r} .
$$

Applying the Lagrange-d'Alembert [13] equations to the composite hand object system (1), (2) the equations of motion can be represented in fingertip coordinates as

$$
\boldsymbol{M}_{r}(\overline{\boldsymbol{x}}) \ddot{\boldsymbol{p}}_{f}+\boldsymbol{C}_{r}(\overline{\boldsymbol{x}}, \dot{\overline{\boldsymbol{x}}}) \dot{\boldsymbol{p}}_{f}+\boldsymbol{g}_{r}(\overline{\boldsymbol{x}})=\boldsymbol{f}_{f}+\boldsymbol{f}_{\text {ext }},
$$

with $\overline{\boldsymbol{x}}=\left(\boldsymbol{q}^{T}, \boldsymbol{x}_{r}^{T}\right)^{T}$ and

$$
\begin{aligned}
\boldsymbol{M}_{r} & =\left(\boldsymbol{G}_{r}^{T} \boldsymbol{M}_{r o}^{-1} \boldsymbol{G}_{r}\right)^{-1}+\boldsymbol{J}_{h}^{-T} \boldsymbol{M}_{h} \boldsymbol{J}_{h}^{-1} \\
\boldsymbol{C}_{r} & =\boldsymbol{G}_{r}^{+M_{r o}} \boldsymbol{C}_{r o} \boldsymbol{G}_{r}^{+M_{r o}{ }^{T}}-\left(\boldsymbol{G}_{r}^{T} \boldsymbol{M}_{r o}^{-1} \boldsymbol{G}_{r}\right)^{-1} \dot{\boldsymbol{G}}_{r} \boldsymbol{G}_{r}^{+M_{r o}{ }^{T}} \\
& +\boldsymbol{J}_{h}^{-T}\left(\boldsymbol{C}_{h} \boldsymbol{J}_{h}^{-1}+\boldsymbol{M}_{h} \dot{\boldsymbol{J}}_{h}^{-1}\right) \\
\boldsymbol{g}_{r} & =\boldsymbol{G}_{r}^{+M_{r o}} \boldsymbol{g}_{r o}+\boldsymbol{J}_{h}^{-T} \boldsymbol{g}_{h} \\
\boldsymbol{f}_{f} & =\boldsymbol{J}_{h}^{-T} \boldsymbol{\tau} .
\end{aligned}
$$

${ }^{4}$ In contrast to [13], the hand Jacobian is defined w. r. t. the inertial frame explaining the independence of $\boldsymbol{x}_{r}$. 
This equation describes the hand object dynamics w.r.t. the Cartesian motion of the fingertips $\boldsymbol{p}_{f} \in \mathbb{R}^{3 N}$ and forms the basis of the damping design. The vector $f_{\text {ext }} \in \mathbb{R}^{3 N}$ represents the external forces acting on the fingertips and the matrix $\boldsymbol{G}_{r}^{+M_{r o}}$ is the dynamically consistent pseudoinverse [14] of the grasp map. In the following the dependence on $q$ will be suppressed for improved readability.

\section{Control Strategies}

The control of a multifingered hand requires on the one hand the control of the pose of the grasped object to be manipulated and equally important the control of the grasping force. Firstly, basic structures for such control laws are discussed. Secondly, the concept and the equations of the IPC are given and a virtual grasp map is defined. A parametrization of the stiffness and a damping design are proposed.

\section{A. Combination of Springs for Object Level Control}

Within the class of impedance based systems two types can be distinguished (compare Fig. 3): The parallel case in which the impedances related to generalized manipulation forces $\boldsymbol{w}_{v o}$ (object spring) and related to internal forces $\boldsymbol{f}_{c}$ (coupling springs) are decoupled. This decoupling requires a mapping of the manipulation forces to the joint torques. Examples are the virtual linkage [7] or the object level impedance control in [6], [10]. A difficult question with this type of controllers is how to distribute the object level force to the fingertips. Often weighted pseudoinverses are used to solve this problem however the physical meaning of the weighting is small. Secondly, the serial case represented e. g. by the IPC [3] in which the object level force acts on a virtual object inertia. This virtual object is furthermore connected with the fingertips via springs, which generate torques in the joints if elongated. In this case the impedances are not decoupled but the force distribution is intuitively given by the choice of the coupling springs. Certainly, the coupling springs in both cases must have different properties: In the parallel case 1D springs are needed which parameterize the internal forces. In the serial case, it is intuitive to adjust the dimensionality of the spring to the contact model at the fingertip. For a PCWF for instance, only forces can be applied to the object. Hence, a translational spring in three dimensions is appropriate. In [10] a control law for the parallel case was proposed and evaluated. Therefore, the next sections will discuss the implementation of a control law with serial structure.

\section{B. IPC with Isotropic Coupling Springs}

In Fig. 4 the structure of the IPC is depicted. The key element of the IPC is the introduction of a virtual object $\boldsymbol{H}_{v}\left(\boldsymbol{x}_{v}\right)$ which is originally connected via spatial coupling springs with the fingertips. It is also connected via another spatial spring (hand configuration spring) with the virtual equilibrium position of the hand $\boldsymbol{H}_{v, d}$ [3]. It is important to note that the coupling springs do not connect with the center of the virtual object but for each coupling spring $i$ a rest

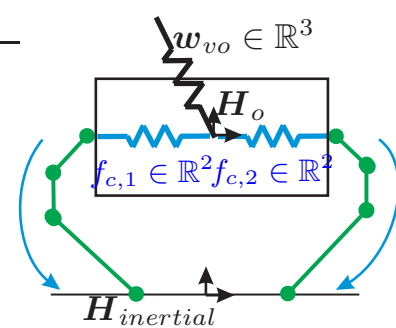

Serial Connection

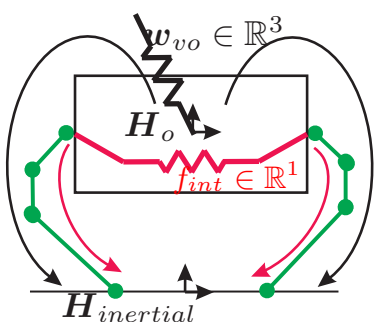

Parallel Connection
Fig. 3. Difference between serial and parallel interconnection of the object level impedances for a planar example. The arrows indicate which forces are mapped directly to the joint torques.

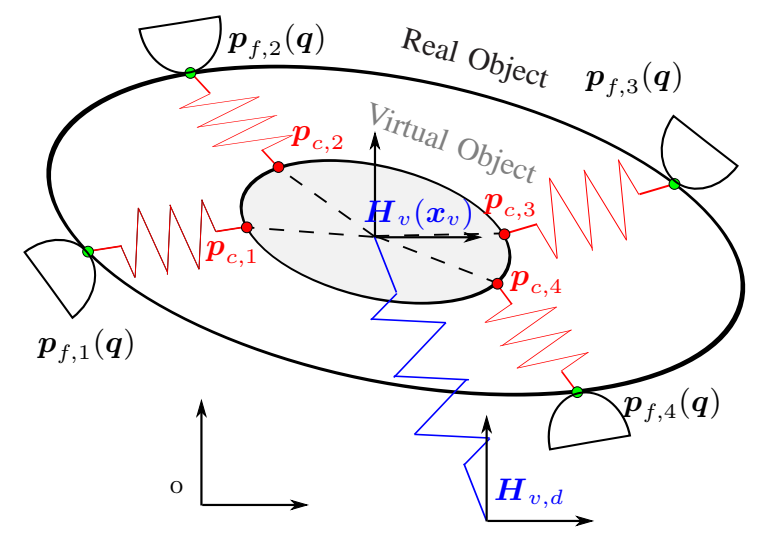

Fig. 4. Virtual object and virtual grasp map for four fingers in contact.

length $\boldsymbol{H}_{v}^{-1} \boldsymbol{H}_{c_{i}}$ is defined. In fact, these transformations can be interpreted as virtual contact points. Table I gives an overview of the used frames. The simulation of the virtual

\begin{tabular}{|c|l|}
\hline $\boldsymbol{H}_{v}$ & Virtual object frame \\
$\boldsymbol{H}_{r}$ & Real object frame \\
$\boldsymbol{H}_{v, d}$ & Desired equilibrium frame of the virtual object \\
$\boldsymbol{H}_{f_{i}}$ & Fingertip frame of finger $i$ \\
$\boldsymbol{H}_{c_{i}}$ & Frame at the $i^{t h}$ virtual contact \\
\hline
\end{tabular}

TABLE I

DEFINITION OF COORDINATE SYSTEMS.

object dynamics can be formulated in the same way as in equation (1)

$$
\boldsymbol{M}_{v}\left(\boldsymbol{x}_{v}\right) \ddot{\boldsymbol{x}}_{v}+\boldsymbol{C}_{v}\left(\boldsymbol{x}_{v}, \dot{\boldsymbol{x}}_{v}\right) \dot{\boldsymbol{x}}_{v}=\boldsymbol{w}_{v},
$$

where the new variables are defined accordingly to equation (1) and the vector $\boldsymbol{w}_{v}$ are the generalized forces applied to the virtual object and represents the control input to the virtual object. In contrast to [3], the coupling springs are chosen to transmit only forces. This choice is motivated by the fact that at the contacts between the robot fingers and the real object only forces can be applied but no torques in case of PCWF. The frames of the virtual fingers $\boldsymbol{H}_{c_{i}}, i=$ $1, \ldots, N$ and the virtual object $\boldsymbol{H}_{v}$ form a grasp that can be described by a virtual grasp map $\boldsymbol{G}_{v}$ that is constructed accordingly to equation (3) replacing the indices $r, f$ with 
$v, c$. The properties described in equation (4) are also valid for the virtual grasp map for the corresponding indices.

The stacked vector $\boldsymbol{f}_{c}$ and the generalized forces $\boldsymbol{w}_{v o}$ are the forces generated by the coupling and the hand configuration spring, respectively. Using equation (4) the effect of the coupling forces at the virtual and the real object can be written as $\boldsymbol{w}_{v c}=\boldsymbol{G}_{v} \boldsymbol{f}_{c}$ and $\boldsymbol{f}_{f}=-\boldsymbol{f}_{c}$. The control inputs $\boldsymbol{w}_{v}=\boldsymbol{w}_{v c}+\boldsymbol{w}_{v o}$ of the virtual object (7) and of the real hand object system $f_{f}(6)$ can be related to the introduced spring forces

$$
\begin{array}{r}
\left(\begin{array}{c}
\boldsymbol{w}_{v} \\
\boldsymbol{f}_{f}
\end{array}\right)=\left(\begin{array}{c}
\boldsymbol{w}_{v c}+\boldsymbol{w}_{v o} \\
\boldsymbol{f}_{f}
\end{array}\right)=\boldsymbol{G}_{D}\left(\begin{array}{c}
\boldsymbol{f}_{c} \\
\boldsymbol{w}_{v o}
\end{array}\right), \\
\boldsymbol{G}_{D}=\left[\begin{array}{cc}
\boldsymbol{G}_{v} & \mathbf{I}_{6 \times 6} \\
-\mathbf{I}_{3 N \times 6} & \mathbf{0}_{3 N \times 6}
\end{array}\right] .
\end{array}
$$

The new control inputs are $\boldsymbol{w}_{v o}, \boldsymbol{f}_{c}$, which are obtained by the compliance control law

$$
\left(\begin{array}{c}
\boldsymbol{f}_{c} \\
\boldsymbol{w}_{v o}
\end{array}\right)=-\frac{\partial V_{s}}{\partial\left(\boldsymbol{p}_{c}^{T}, \boldsymbol{x}_{v}^{T}\right)^{T}}-\left(\begin{array}{c}
\boldsymbol{f}_{c d} \\
\boldsymbol{w}_{v o d}
\end{array}\right),
$$

with $\boldsymbol{f}_{c d}, \boldsymbol{w}_{\text {vod }}$ being the damping terms parallel to the coupling and the hand configuration springs. The hand configuration and the coupling springs can be defined by the superposition of their potential functions

$$
V_{s}=V_{o}\left(\boldsymbol{x}_{v}, \boldsymbol{x}_{v, d}, \boldsymbol{K}_{o}\right)+V_{c}\left(\boldsymbol{p}_{f}, \boldsymbol{p}_{c}, \boldsymbol{K}_{c}\right) .
$$

Energy functions for the spatial spring connecting the virtual object with the virtual equilibrium frame $\boldsymbol{H}_{v, d}$, which are explained in detail in [15] and [5] are used

$$
V_{o}\left(\boldsymbol{x}_{v}, \boldsymbol{x}_{v, d}, \boldsymbol{K}_{o}\right)=\frac{1}{2}\left(\boldsymbol{x}_{v}-\boldsymbol{x}_{v, d}\right)^{T} \underbrace{\boldsymbol{K}_{o}\left(\boldsymbol{x}_{v}-\boldsymbol{x}_{v, d}\right)}_{\boldsymbol{w}_{v o, k}},
$$

with $\boldsymbol{K}_{o}$ the symmetric and positive definite (p. d.) object stiffness matrix and the object spring generalized force $\boldsymbol{w}_{v o, k}$. The potential function of the coupling springs

$$
V_{c}\left(\boldsymbol{p}_{f}, \boldsymbol{p}_{c}, \boldsymbol{K}_{c}\right)=\frac{1}{2}\left(\boldsymbol{p}_{c}-\boldsymbol{p}_{f}\right)^{T} \underbrace{\boldsymbol{K}_{c}\left(\boldsymbol{p}_{c}-\boldsymbol{p}_{f}\right)}_{\boldsymbol{f}_{k}}
$$

is parameterized by $\boldsymbol{p}_{c}$ and the p. d. and symmetric coupling stiffness matrix $\boldsymbol{K}_{c}=\operatorname{blockdiag}\left\{k_{c_{1}} \mathbf{I}_{3 \times 3}, \ldots, k_{c_{N}} \mathbf{I}_{3 \times 3}\right\}$. The vector $\boldsymbol{f}_{k}$ contains the stacked coupling spring forces of all fingers. Since only isotropic springs can be purely translational [3], they were chosen for the coupling springs.

Inserting the potential function (10) into the compliance control law (9), and using equation (4) $\delta \boldsymbol{p}_{c}=\boldsymbol{G}_{v}^{T} \delta \boldsymbol{x}_{v}$ we obtain

$$
\begin{aligned}
\left(\begin{array}{c}
\boldsymbol{f}_{c} \\
\boldsymbol{w}_{v o}
\end{array}\right) & =-\overline{\boldsymbol{K}} \boldsymbol{G}_{D}^{T}\left(\begin{array}{c}
\boldsymbol{x}_{v} \\
\boldsymbol{p}_{f}
\end{array}\right)-\left(\begin{array}{c}
\boldsymbol{f}_{c d} \\
\boldsymbol{w}_{v o d}
\end{array}\right)-\boldsymbol{w}_{d o} \\
\overline{\boldsymbol{K}} & =\left[\begin{array}{cc}
\boldsymbol{K}_{c} & \mathbf{0} \\
\mathbf{0} & \boldsymbol{K}_{o}
\end{array}\right] \boldsymbol{w}_{d o}=\left(\begin{array}{c}
\mathbf{0} \\
\boldsymbol{K}_{o} \boldsymbol{x}_{v, d}
\end{array}\right)(13)
\end{aligned}
$$

It is important to note that the applied mapping of equation (4) is only locally valid and hence the derived control law. Now, we can combine the equations of motion of the virtual object (7) and the composite real object robot hand system
(6) and insert the control inputs $\boldsymbol{f}_{c}, \boldsymbol{w}_{v o}$ using equations (13) and (8). The closed loop dynamics with the corresponding state vector $\boldsymbol{x}=\left[\boldsymbol{x}_{v}^{T} \boldsymbol{p}_{f}^{T}\right]^{T}$ can be then written as

$$
\begin{array}{cc}
\boldsymbol{M} \ddot{\boldsymbol{x}}+\mathbf{C G}+\boldsymbol{G}_{D}\left(\begin{array}{c}
\boldsymbol{f}_{c d} \\
\boldsymbol{w}_{\text {vod }}
\end{array}\right)+\boldsymbol{K} \boldsymbol{x}=\boldsymbol{w}, \\
\boldsymbol{M}=\left[\begin{array}{cc}
\boldsymbol{M}_{v} & \mathbf{0} \\
\mathbf{0} & \boldsymbol{M}_{r}
\end{array}\right] \quad \mathbf{C G}=\left(\begin{array}{c}
\boldsymbol{C}_{v} \\
\boldsymbol{C}_{r}+\boldsymbol{g}_{r}
\end{array}\right) \\
\boldsymbol{K}=\boldsymbol{G}_{D} \overline{\boldsymbol{K}} \boldsymbol{G}_{D}^{T} & \boldsymbol{w}=\left(\begin{array}{c}
\boldsymbol{K}_{o} \boldsymbol{x}_{v, d} \\
\boldsymbol{f}_{\text {ext }}
\end{array}\right) .
\end{array}
$$

In order to apply the control law (13) to the robot, the coupling forces have to be mapped into the joint space using the transposed hand Jacobian

$$
\boldsymbol{\tau}=\boldsymbol{J}_{h}^{T}(\boldsymbol{q}) \boldsymbol{f}_{f} .
$$

\section{Requirements on the virtual grasp map $\boldsymbol{G}_{v}$}

In [3] and [4], the coupling springs between the virtual object and the robot end-effectors were designed as spatial springs that transmit forces as well as torques. This is analog to a rigid grasp, where one end-effector is sufficient to form a stable grasp. The steady-state solution of the equations of motion of the virtual object in equation (14) together with $\boldsymbol{w}_{\text {ext }}=\mathbf{0}$ gives

$$
\boldsymbol{w}_{v o}=-\boldsymbol{G}_{v} \boldsymbol{f}_{c} .
$$

From this equation it is obvious that the virtual grasp map $\boldsymbol{G}_{v}$ has to have full row rank. An important property of a grasp is force closure: If and only if $\boldsymbol{G}_{v}(\mathbf{F C})=\mathbb{R}^{6}$ with $\mathbf{F C}=\mathbf{F C}_{1} \times \cdots \times \mathbf{F} \mathbf{C}_{N} \subset \mathbb{R}^{3 N}$ a grasp is force closure.

\section{Choice of Stiffness Parameters}

The IPC realizes a serial connection of the object and the coupling springs from $\boldsymbol{H}_{v, d}$ to $\boldsymbol{H}_{r}$ assuming that the object is rigidly connected to the real fingertips. From an application point of view it is desirable to be able to define the effective stiffness $\boldsymbol{K}_{\text {eff }} \in \mathbb{R}^{6 \times 6}$ for the real object, i. e. the change in pose due to an external wrench

$$
\boldsymbol{K}_{\text {eff }} \boldsymbol{x}_{r}=\boldsymbol{w}_{\text {ext }} .
$$

The steady-state of the closed-loop dynamics (14) in the coordinates of the real and the virtual object $\boldsymbol{x}_{v r}=\left[\boldsymbol{x}_{v}^{T} \boldsymbol{x}_{r}^{T}\right]^{T}$ is given by

$$
\begin{aligned}
& \boldsymbol{G}_{E} \overline{\boldsymbol{K}} \boldsymbol{G}_{E}^{T} \boldsymbol{x}_{v r}=\overline{\boldsymbol{w}} \\
& \text { with } \quad \boldsymbol{G}_{E}=\left[\begin{array}{cc}
\boldsymbol{G}_{v} & \mathbf{I} \\
-\boldsymbol{G}_{r} & \mathbf{0}
\end{array}\right] \text { and } \overline{\boldsymbol{w}}=\left[\begin{array}{c}
\delta \boldsymbol{x}_{v, d} \\
\boldsymbol{w}_{\text {ext }}
\end{array}\right]
\end{aligned}
$$

while neglecting the gravity term and using equation (4). A reasonable method to achieve an effective stiffness is to set $\boldsymbol{K}_{c}, \boldsymbol{G}_{r}, \boldsymbol{G}_{v}$ depending on the task and to solve for $\boldsymbol{K}_{o}$. Equation (18) can be also written as

$$
\left[\begin{array}{cc}
\boldsymbol{K}_{1} & \boldsymbol{K}_{12} \\
\boldsymbol{K}_{12}^{T} & \boldsymbol{K}_{2}
\end{array}\right]\left(\begin{array}{l}
\boldsymbol{x}_{v} \\
\boldsymbol{x}_{r}
\end{array}\right)=\overline{\boldsymbol{w}} .
$$

Solving this equation for $\boldsymbol{x}_{r}$ and $\boldsymbol{x}_{v}$ we can easily identify the matrix $\boldsymbol{K}_{\text {eff }}$.

$$
\boldsymbol{K}_{\text {eff }}=\boldsymbol{K}_{2}-\boldsymbol{K}_{12}^{T} \boldsymbol{K}_{1}^{-1} \boldsymbol{K}_{12} .
$$


Finally, using this equation together with equation (18) the stiffness for the hand configuration spring is obtained

$$
\boldsymbol{K}_{o}=\boldsymbol{K}_{12}\left(\boldsymbol{K}_{2}-\boldsymbol{K}_{e f f}\right)^{-1} \boldsymbol{K}_{12}^{T}-\boldsymbol{G}_{v} \boldsymbol{K}_{c} \boldsymbol{G}_{v}^{T} .
$$

Notice, that the effective stiffness of $\boldsymbol{K}_{c}$ represents an upper bound to the achievable effective stiffness $\boldsymbol{K}_{\text {eff } f}$.

\section{E. Damping Design}

Obviously, $\boldsymbol{K}, \boldsymbol{M} \in \mathbb{R}^{(6+3 N) \times(6+3 N)}$ are symmetric. With these matrices, the neglect of the Coriolis and the gravity terms, setting $\boldsymbol{x}_{v, d}=\mathbf{0}$, and a quasi-static consideration the closed loop dynamical equations (14) can be written as

$$
M \ddot{x}+D \dot{x}+K x=0,
$$

where the damping term

$$
\boldsymbol{G}_{D}\left(\begin{array}{c}
\boldsymbol{f}_{c d} \\
\boldsymbol{w}_{\text {vod }}
\end{array}\right)=\boldsymbol{D} \dot{\boldsymbol{x}}
$$

is replaced. This brings equation (22) in the form of a classical mass-spring-damper system. Because of the symmetry of $K$ and the symmetric and p. d. matrix $\boldsymbol{M}$ the damping design using double-diagonalization [16] can be then applied.

From the matrix pair $(\boldsymbol{K}, \boldsymbol{M})$ we obtain a damping matrix $D$. The term $\xi$ represents the damping coefficient and parameterizes the damping term. The desired damping forces have to equal the damping terms which are injected by equation (13). Hence, equation (23) has to be solved for $\left(\boldsymbol{f}_{c d}^{T}, \boldsymbol{w}_{v o d}^{T}\right)^{T}$. The inverse of the matrix $\boldsymbol{G}_{D} \in \mathbb{R}^{(6+3 N) \times(6+3 N)}$ is therefore needed. If the virtual and the real grasp matrix realize stable grasps $G_{D}$ has full rank, and we obtain

$$
\left(\begin{array}{c}
\boldsymbol{f}_{c d} \\
\boldsymbol{w}_{v o d}
\end{array}\right)=\boldsymbol{G}_{D}^{-1} \boldsymbol{D} \dot{\boldsymbol{x}}
$$

Since $\dot{\boldsymbol{x}}_{r}$ cannot be measured directly, the Moore-Penrose pseudoinverse of the transposed virtual grasp map ${ }^{5}$ is used to calculate the velocity of the real object from the velocities of the fingertips

$$
\dot{\boldsymbol{x}}_{r}=\boldsymbol{G}_{r}^{T+} \dot{\boldsymbol{p}}_{f} .
$$

Furthermore, in the real-time implementation it is easier to integrate equation (25) over time to obtain $\boldsymbol{x}_{r}$, respectively $\boldsymbol{H}_{r}\left(\boldsymbol{x}_{r}\right)$, instead of simulating the real object dynamics.

\section{EXPERIMENTS}

The proposed implementation of the IPC including the damping design has been integrated on the DLR Hand II [12]. In Fig. 1 the hand is superimposed by the virtual object and the springs attached to it. The hand has four fingers with 3 DOF each, resulting in overall 12 DOF. In addition to the position sensors the link-side torques are measured as well. The control law runs on a QNX target on a Pentium IV with $3 \mathrm{GHz}$ with a controller sample time of $1 \mathrm{~ms}$. The desired control torque from equation (15) is used as set point for a low-level torque controller which is based on the joint torque

\footnotetext{
${ }^{5}$ In this case the left pseudoinverse is used that is independent of its weighting.
}
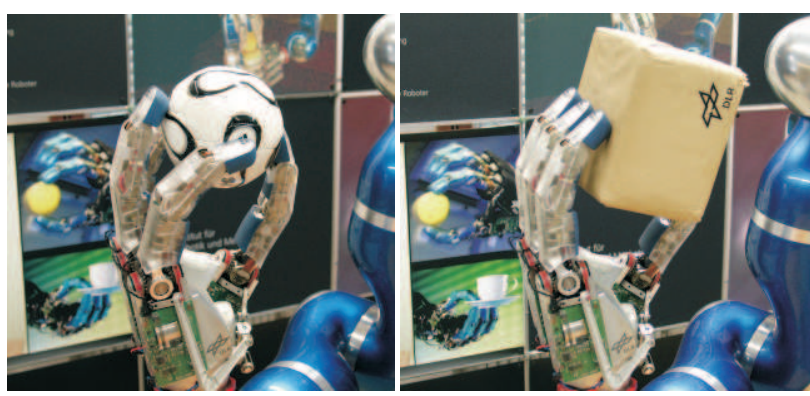

Fig. 5. The DLR Hand II grasping a toy ball (left) and a box (right).

measurement, and offline estimated static and viscous motor friction parameters.

The control law is evaluated in two experiments (compare Fig. 5): A) Object rotation and translation of a soft toy ball with a diameter of $0.11 \mathrm{~m}$ and a weight of $0.1 \mathrm{~kg}$. B) Object translation of a stiff box with dimensions $0.14 m \times$ $0.14 \mathrm{~m} \times 0.1 \mathrm{~m}$ and a weight of $0.2 \mathrm{~kg}$. For the experiments the controller parametrization is given in Table II. The used parameters lead to the effective translational stiffness $\boldsymbol{K}_{\text {eff,t }}=\operatorname{diag}\{705,705,705\} \mathrm{N} / \mathrm{m}$ for both experiments and $\boldsymbol{K}_{\text {eff,r }}=\operatorname{diag}\{2.5,2.9,4.7\} \mathrm{Nm} / \mathrm{rad}$ for the ball and $\boldsymbol{K}_{\text {eff,r }}=\operatorname{diag}\{1.6,2.0,3.3\} \mathrm{Nm} / \mathrm{rad}$ for the box experiment. Note that assumption 3) in section II introduces a small kinematic error since the fingertips have a round shape.

\begin{tabular}{|c|c|c|}
\hline $\boldsymbol{K}_{t}[N / m]$ & $\boldsymbol{K}_{r}[N m / r a d]$ & $\boldsymbol{K}_{c}[N / m]$ \\
\hline $\operatorname{diag}\{1000,1000,1000\}$ & $\operatorname{diag}\{20,20,20\}$ & $600 \mathbf{I}_{3 N \times 3 N}$ \\
\hline \hline$m_{v}[\mathrm{~kg}]$ & $\mathbf{I}_{v}\left[\mathrm{kgm}^{2}\right]$ & $\xi$ \\
\hline 0.1 & $0.003 \mathbf{I}_{3 \times 3}$ & 1 \\
\hline
\end{tabular}

TABLE II

CONTROLLER PARAMETERS.

\section{A. Manipulation of a soft Object: Toy Ball}

The grasped ball is translated along the $y$-axis by commanding a step of $0.04 \mathrm{~m}$. The step response of the object spring force $\boldsymbol{f}_{v o, k}$ is presented in Fig. 6 . The response converges after $250 \mathrm{~ms}$ well damped to a steady-state. The remaining error is less than $5 \mathrm{~N}$ which stems from joint friction ${ }^{6}$. In Fig. 7 we observe changes of the object spring torque $\boldsymbol{m}_{v o, k}$ in the magnitude of $0.2 \mathrm{Nm}$ during the translation indicating that the coupling between rotation and translation is small. In Fig. 8 the coupling forces of finger $2 \boldsymbol{f}_{k, 2}$ have an offset which represents internal forces and the transient behavior is well damped as well. The difference between virtual object and real object position $\Delta \boldsymbol{p}=\boldsymbol{p}_{v}-\boldsymbol{p}_{r}$ is printed in Fig. 9 showing the transient behavior of the relative motion of the objects. Initially, the difference is small which then grows to a maximum of $0.008 \mathrm{~m}$ corresponding to $20 \%$ of the step size. At the moment of maximum error the virtual object reached the desired value and the real

\footnotetext{
${ }^{6}$ The friction compensation in the torque controller is chosen rather conservative to ensure stability.
} 
object is basically pulled then only by the coupling springs to its steady-state. In another experiment the grasped ball is

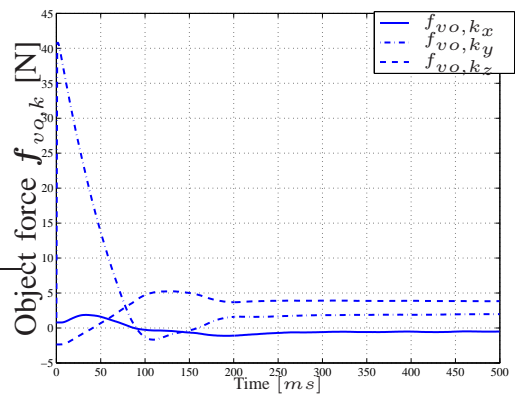

Fig. 6. Object spring force $\boldsymbol{f}_{v o, k}$ during the translation along $y$-axis of $0.04 \mathrm{~m}$ (Ball).

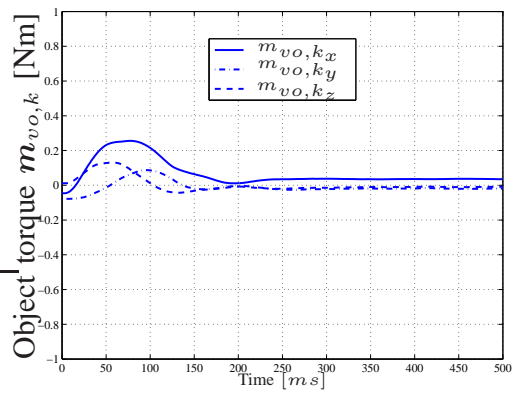

Fig. 7. Object spring torque $\boldsymbol{m}_{v o, k}$ during the translation along $y$-axis of $0.04 \mathrm{~m}$ (Ball).

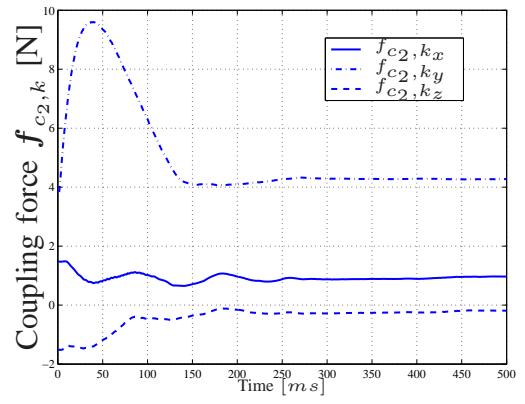

Fig. 8. Coupling spring force $\boldsymbol{f}_{k, 2}$ during the translation along $y$-axis of $0.04 \mathrm{~m}$ (Ball).

rotated around the $z$-axis by commanding a step of $0.6 \mathrm{rad}$. The step response of the object spring torque $\boldsymbol{m}_{v o, k}$ shows in Fig. 10 a well damped convergence. Fig. 11 indicates small coupling with the translational motions. Fig. 12 depicts the coupling forces of finger 2 . Since these forces are depicted in the inertial frame the internal forces converge to different steady-state values.

\section{B. Manipulation of a stiff Object: Box}

In this experiment the controller was tested to manipulate a stiff box which has virtually no intrinsic damping.

The grasped box is translated along the $y$-axis by commanding a step $0.04 \mathrm{~m}$. The step response of the object spring

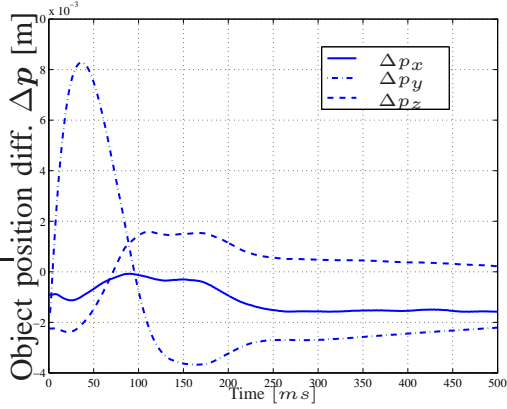

Fig. 9. Difference between virtual object and real object position $\boldsymbol{p}_{v}-\boldsymbol{p}_{r}$ during the translation along $y$-axis of $0.04 \mathrm{~m}$ (Ball).

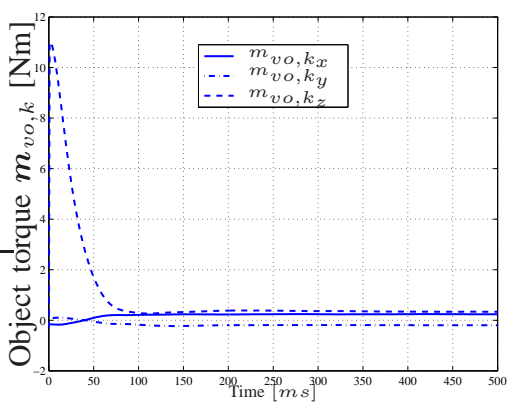

Fig. 10. Object spring torque $\boldsymbol{m}_{v o, k}$ during the rotation around $y$-axis of $0.6 \mathrm{rad}($ Ball $)$

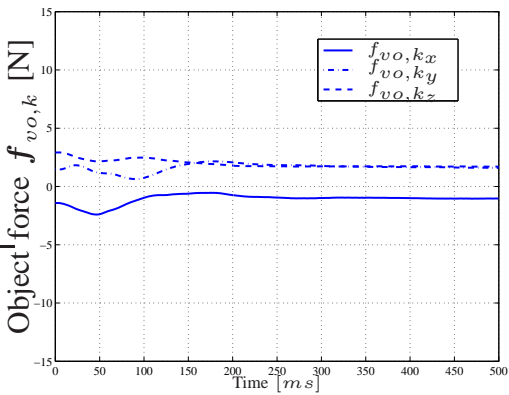

Fig. 11. Object spring force $\boldsymbol{f}_{v o, k}$ during the rotation around $y$-axis of $0.6 \mathrm{rad}$ (Ball).

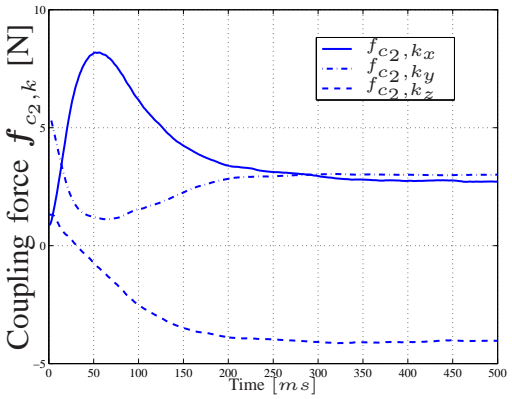

Fig. 12. Coupling spring force $\boldsymbol{f}_{k, 2}$ during the rotation around $y$-axis of $0.6 \mathrm{rad}$ (Ball).

force $\boldsymbol{f}_{v o, k}$ is presented in Fig. 13. The response converges after $250 \mathrm{~ms}$ well damped to a steady-state. In Fig. 14 a small 
coupling between the translational and the rotational motion can be observed. In Fig. 15 the coupling forces of finger $2 \boldsymbol{f}_{k, 2}$ are converging to a steady-state. Note that since the box has no intrinsic damping it is very important to inject damping w.r.t. the internal motions by means of the controller (compare equation (24)).

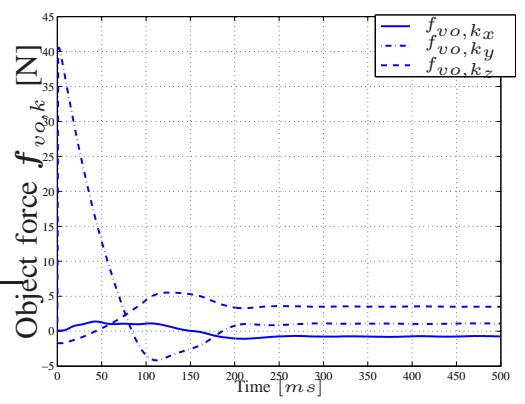

Fig. 13. Object spring force $\boldsymbol{f}_{v o, k}$ during the translation along $y$-axis of $0.04 \mathrm{~m}$ (Box).

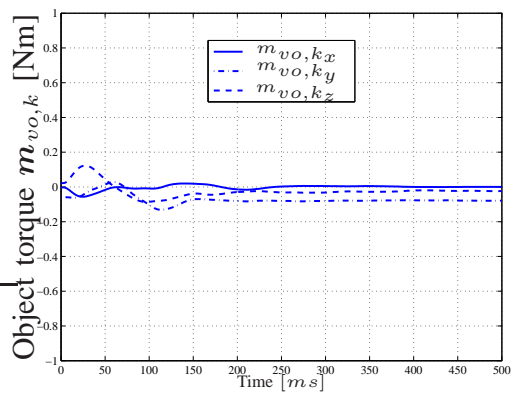

Fig. 14. Object spring torque $\boldsymbol{m}_{v o, k}$ during the translation along $y$-axis of $0.04 \mathrm{~m}$ (Box).

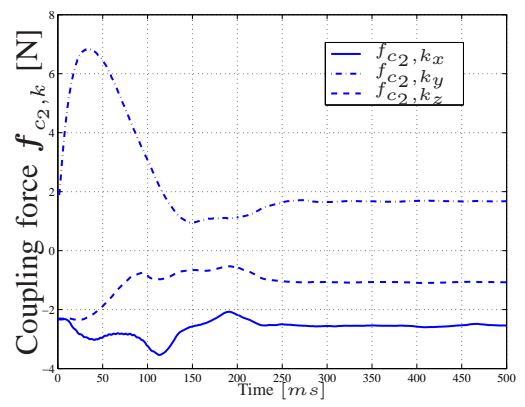

Fig. 15. Coupling spring force $\boldsymbol{f}_{k, 2}$ during the translation along $y$-axis of $0.04 \mathrm{~m}$ (Box).

\section{CONCLUSIONS}

An implementation of the IPC originally presented by Stramigioli [3] was analyzed. Instead of spatial coupling springs, isotropic coupling springs that transmit only force components were employed. The difference between dexterous hand control laws realizing a parallel [10] and a serial interconnection of hand configuration and coupling springs was discussed. In this paper the focus was on the implementation and evaluation of a serial structure. The term virtual grasp map was introduced and the necessary conditions of its force closure were stated. A method to parameterize the stiffness parameters in order to obtain an effective object level stiffness and a damping design was proposed. The controller was implemented on the DLR Hand II and its performance was analyzed through step commands manipulating soft and stiff objects. The controller produced well-damped responses w.r.t. object motion but also w.r.t. to internal motions. Future work will be to compare the performance and practicability of the serial with the parallel connection of springs through experiments.

\section{REFERENCES}

[1] S. A. Schneider and J. R. H. Cannon, "Object impedance control for cooperative manipulation: Theory and experimental results," IEEE Transactions on Robotics and Automation, vol. 8, no. 3, pp. 383-394, 1992.

[2] A. Bicchi, "Hand for dexterous manipulation and robust grasping: A difficult road towards simplicity," IEEE Transactions on Robotics and Automation, vol. 16, no. 6, pp. 652-662, 2000.

[3] S. Stramigioli, Modeling and IPC Control of Interactive Mechanical Systems: A Coordinate-free Approach, ser. Lecture Notes in Control and Information Sciences. Springer-Verlag, 2001, vol. 266.

[4] C. Secchi, S. Stramigioli, and C. Melchiorri, "Geometric grasping and telemanipulation," in IEEE/RSJ International Conference on Intelligent Robots and Systems, 2001, pp. 1763-1768.

[5] C. Natale, Interaction Control of Robot Manipulators: Six-Degreesof-Freedom Tasks, ser. Springer Tracts in Advanced Robotics (STAR). Springer-Verlag, 2003, vol. 3.

[6] T. Wimböck, Ch. Ott, and G. Hirzinger, "Impedance behaviors for twohanded manipulation: Design and experiments," in IEEE International Conference on Robotics and Automation, 2007, pp. 4182-4189.

[7] D. Williams and O. Khatib, "The virtual linkage: A model for internal forces in multi-grasp manipulation," in IEEE International Conference on Robotics and Automation, 1993, pp. 1025-1030.

[8] S. Arimoto, J.-H. Bae, and K. Tahara, "Stability on a manifold: Simultaneous realization of grasp and orientation control of an object by a pair of robot fingers," in IEEE International Conference on Robotics and Automation, 2003, pp. 2336-2343.

[9] R. G. Bonitz and T. Hsia, "Internal force-based impedance control for cooperating manipulators," IEEE Transactions on Robotics and Automation, vol. 12, pp. 78-89, 1996.

[10] T. Wimböck, Ch. Ott, and G. Hirzinger, "Passivity-based objectlevel impedance control for a multifingered hand," in IEEE/RSJ International Conference on Intelligent Robots and Systems, 2006, pp. $4621-4627$.

[11] Ch. Ott, O. Eiberger, W. Friedl, B. Bäuml, U. Hillenbrand, Ch. Borst, A. Albu-Schäffer, B. Brunner, H. Hirschmüller, S. Kielhöfer, R. Konietschke, M. Suppa, T. Wimböck, F. Zacharias, and G. Hirzinger, "A humanoid two-arm system for dexterous manipulation," in IEEE-RAS International Conference on Humanoid Robots, Genova/Italy, 2006 , pp. 276-283.

[12] J. Butterfaß, M. Grebenstein, H. Liu, and G. Hirzinger, "DLR-Hand II: Next generation of a dextrous robot hand," in IEEE International Conference on Robotics and Automation, 2001.

[13] R. Murray, Z. Li, and S. Sastry, A Mathematical Introduction to Robotic Manipulation. CRC Press, 1994.

[14] O. Khatib, "Inertial properties in robotic manipulation: An object-level framework," International Journal of Robotics Research, vol. 14, no. 1 , pp. 19-36, 1995

[15] F. Caccavale, C. Natale, B. Siciliano, and L. Villani, "Six-dof impedance control based on angle/axis representations," IEEE Transactions on Robotics and Automation, vol. 15, no. 2, pp. 289-299, 1999.

[16] A. Albu-Schäffer, Ch. Ott, and G. Hirzinger, "A passivity based cartesian impedance controller for flexible joint robots - part II: Full state feedback, impedance design and experiments," in IEEE International Conference on Robotics and Automation, 2004, pp. 2666-2672. 